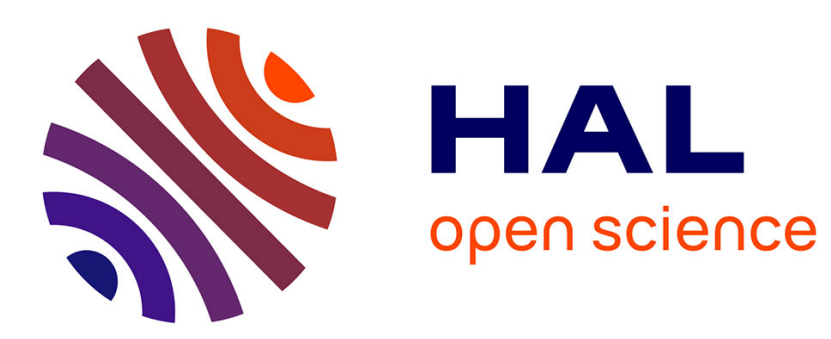

\title{
Inverse Localization of Ischemia in a 3D Realistic Geometry: A Level Set Approach
}

Carlos E. Chavez, Felipe Alonso-Atienza, Nejib Zemzemi, Yves Coudière, Diegó Alvarez

\section{To cite this version:}

Carlos E. Chavez, Felipe Alonso-Atienza, Nejib Zemzemi, Yves Coudière, Diegó Alvarez. Inverse Localization of Ischemia in a 3D Realistic Geometry: A Level Set Approach. Computing in cardiology, Sep 2015, Nice, France. hal-01241754

\section{HAL Id: hal-01241754 \\ https://hal.inria.fr/hal-01241754}

Submitted on 10 Dec 2015

HAL is a multi-disciplinary open access archive for the deposit and dissemination of scientific research documents, whether they are published or not. The documents may come from teaching and research institutions in France or abroad, or from public or private research centers.
L'archive ouverte pluridisciplinaire HAL, est destinée au dépôt et à la diffusion de documents scientifiques de niveau recherche, publiés ou non, émanant des établissements d'enseignement et de recherche français ou étrangers, des laboratoires publics ou privés. 


\title{
Inverse Localization of Ischemia in a 3D Realistic Geometry: A Level Set Approach
}

\author{
Carlos E Chávez ${ }^{1}$, Felipe Alonso-Atienza ${ }^{2}$, Nejib Zemzemi ${ }^{3,4}$, Yves Coudière ${ }^{3,4}$, Diego Álvarez ${ }^{1}$ \\ ${ }^{1}$ University Carlos III of Madrid, Leganés, Spain \\ ${ }^{2}$ University Rey Juan Carlos, Fuenlabrada, Spain \\ ${ }^{3}$ INRIA Bordeaux - Soud-Ouest, Bordeaux, France \\ ${ }^{4}$ Electrophysiology and Heart Modeling Institute (IHU LIRYC), Bordeaux, France
}

\begin{abstract}
The reconstruction of cardiac ischemic regions from body surface potential measurements (BSPMs) is usually performed at a single time instant which corresponds to the plateau or resting phase of the cardiac action potential. Using a different approach, we previously proposed a level set formulation that incorporates the knowledge of the cardiac excitation process in the inverse procedure, thus exploiting the spatio-temporal correlations contained in the BSPMs. In this study, we extend our inverse levelset formulation for the reconstruction of ischemic regions to $3 D$ realistic geometries, and analyze its performance in different noisy scenarios. Our method is benchmarked against zero-order Tikhonov regularization. The inverse reconstruction of the ischemic region is evaluated using the correlation coefficient (CC), the sensitive error ratio $(S N)$, and the specificity error ratio (SP). Our algorithm outperforms zero-order Tikhonov regularization, specially in highly noisy scenarios.
\end{abstract}

\section{Introduction}

The inverse problem of electrocardiography (IPE) aims to characterize the cardiac electric sources (membrane potential, epicardial or endocardical potentials, activation times) and/or the cardiac substrate (ischemia, post-infarct scars) regions from body surface potentials measurements (BSPMs) [1]. The IPE is a hard technological challenge since in its general formulation is an ill-posed problem so a number of regularization approaches have been developed over the years to obtain stable and realistic solutions $[2,3]$.

Several works have analyzed the the IPE in terms of localizing cardiac ischemic regions [4-7]. In these studies the ischemic regions are assessed by reconstructing the transmembrane or the epicardial potentials at a single time-instant during the plateau phase of the action poten- tial, thus ignoring the spatiotemporal correlation information contained in the BSPMs. Using a different approach, we previously developed an inverse procedure that exploits the spatio-temporal correlations contained in the BSPMs through a mathematical model that describes the electrical activity of the heart [8].

In this work, we extended our methodology defined in [8] to 3D realistic anatomical models. The performance of the inverse procedure was analyzed in different noisy scenarios, where BSPMs were corrupted by AWGN with different signal-to-noise ratios (SNRs). Our method was benchmarked against zero-order Tikhonov regularization. Results showed that our algorithm outperformed zeroorder Tikhonov regularization, specially in highly noisy scenarios (low SNRs).

\section{Forward Model}

\subsection{Realistic anatomical Model}

A 3D realistic anatomical model was obtained from the segmentation and discretization of CT scan images of a 43 years old woman. The resulting geometry model consisted of triangular meshes of 5842 and 5742 nodes, for the heart and the torso domains, respectively (figure 1, panels (a) and (b)).

\subsection{Model of action potential}

Cardiac electrical activity at the cell level was simulated by a modified version of the two-current model (TC) [9], which is formulated using two variables: the transmembrane potential $v(t)$, and the inactivation gate variable $h(t)$. The voltage, which is dimensionless and varies between zero and one, is defined as follows

$$
\frac{d v}{d t}=J_{T C}=J_{\text {stim }}(t)+J_{\text {in }}(v, h)+J_{\text {out }}(v),
$$




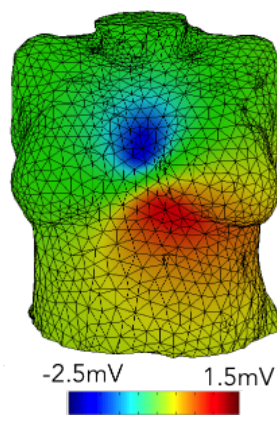

(a)

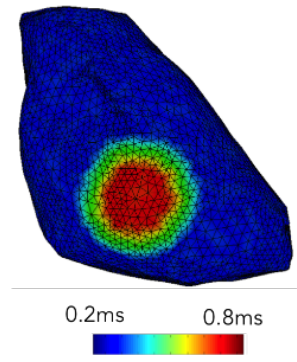

(b)

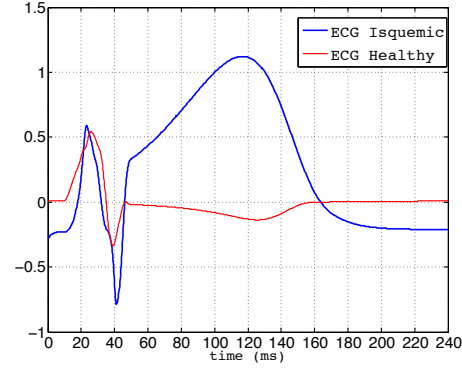

(c)

Figure 1. Forward model. (a) Snapshot of cardiac activation at the torso geometry at time $30 \mathrm{~ms}$. (b) Heart geometry with an ischemic region ( ${ }_{i n}\left(\mathbf{r}_{H}\right)$ profile). (c) Resulting ECG at a single recording site, both for ischemic a healthy conditions.

where $J_{\text {stim }}$ represents the initial stimulus, $J_{\text {in }}$ and $J_{\text {out }}$ denotes the sum of all inward and outward currents, respectively, which are defined as

$$
\begin{gathered}
J_{\text {in }}(v, h)=\frac{h(1-v)\left(v-v_{\text {rest }}\right)^{2}}{i n}, \\
J_{\text {out }}(v, h)=-\frac{v-v_{\text {rest }}}{\text { out }},
\end{gathered}
$$

where $v_{\text {rest }}$ is the resting potential, which was incorporated in [8] to simulate the increase of the resting potential during ischemia.

The gating variable $h(t)$ is also dimensionless and varies between zero and one. This variable regulates inward current flows and obeys the following equation

$$
\frac{d h}{d t}=\begin{array}{clr}
(1-h) / \text { open }, & v<v_{\text {crit }} \\
-h / \text { close }, & v & v_{\text {crit }}
\end{array}
$$

where $v_{\text {crit }}$ is the change-over voltage. This model contains four time constants ( in, out, open and close) which correspond to the four phases of the cardiac action potential: initiation, plateau, decay and recovery.

The effects of ischemia were simulated by modifying the values of in and $v_{\text {rest }}$ [10]. For ischemic cells, we set the parameter in and $v_{r e s t}$ equal to $0.8 \mathrm{~ms}$ and 0.1 , respectively. For healthy cells in and $v_{\text {rest }}$ were set to $0.2 \mathrm{~ms}$ and 0 , respectively.

\subsection{Model of cardiac tissue}

Let $H \quad \mathbb{R}^{3}$ be the cardiac tissue, and $v=v\left(\mathbf{r}_{H}, t\right)$ the membrane potential with $\mathbf{r}_{H} \quad H$. The propagation of $v$ is described according to the monodomain formalism,

$$
\frac{v}{t}=D \cdot(v)+J_{T C}
$$

where $J_{T C}$ is the ion current term current provided by TC model, and $D$ is the intracellular conductivity tensor (assumed constant $D=1.4 \cdot I \mathrm{~mm}^{2} / \mathrm{ms}$ ). Equation (5) is solved by imposing the initial conditions $v=v_{\text {rest }}$ at $t=0$, and no-flux boundary conditions.

To simulate the effects of ischemia at the tissue level, we considered a regional model of ischemia where the parameters in $\left(\mathbf{r}_{H}\right), v_{\text {rest }}\left(\mathbf{r}_{H}\right)$ vary linearly between healthy and ischemic values $[8,11]$. Figure 1 (b) shows the ${ }_{\text {in }}\left(\mathbf{r}_{H}\right)$ profile that represents the ischemic region at the heart surface.

\subsection{Model of BPSMs}

The resulting potential distribution at position $\mathbf{r}_{\mathbf{T}} \quad T$ outside the cardiac tissue $H$, was calculated as

$$
\left(\mathbf{r}_{T}, t\right)=\frac{1}{40}{ }_{H} \frac{D \cdot\left(v\left(\mathbf{r}_{H}, t\right)\right)}{R\left(\mathbf{r}_{H}, \mathbf{r}_{T}\right)} d{ }_{H}
$$

where $R\left(\mathbf{r}_{H}, \mathbf{r}_{T}\right)=\left\|\mathbf{r}_{T}-\mathbf{r}_{H}\right\|$ represents the distance from the source location point $\mathbf{r}_{H}$ to the observation point $\mathbf{r}_{T}, \quad{ }_{0}$ is the medium conductivity (assumed homogeneous and set to $1 \mathrm{~S} / \mathrm{m})$, and $v\left(\mathbf{r}_{H}, t\right)$ is solution of (5).

Using finite element methods, equation (6) can be extended to $N$ recording sites

$$
(t)=\mathbf{A} \cdot \mathbf{v}(t)
$$

where $\mathbf{A}$ is the so-called transfer matrix that linearly relates transmembrane potential distribution $\mathbf{v}(t)$ to the torso potentials $(t)$. Figure 1 (c) represents the resulting BSPMs at a single recording site for both healthy (no ischemia was present) and ischemic conditions.

\section{Inverse procedures}

\subsection{Proposed formulation}

Let $H$ be a synthetic cardiac tissue which contains an ischemic region $\mathcal{S} \quad H$, and ${ }_{R}(t)$ be the observed BSPM, with $t \quad[0, T]$, being $T$ the total recording time. 
Ischemic regions were estimated by considering a binary output: healthy and ischemic tissues. This way, a shape reconstruction problem can be formulated using a level set function to define the spatial distribution of in and $v_{\text {rest }}$ parameters, so that

$$
\left(\mathbf{r}_{H}\right)=\begin{array}{rlll}
0 & \text { if } & \mathbf{r}_{H} & \mathcal{S} \\
0 & \text { if } & \mathbf{r}_{H} / \mathcal{S}
\end{array}
$$

However, since both parameters, define the same region, we only considered the variation of $i n$.

In order to recover the ischemic region, we adjusted $\left(\mathbf{r}_{H}\right)$ by minimizing the misfit between ${ }_{R}(t)$, and the BSPM associated to a guess configuration ${ }_{S}\left(t ;{ }_{i n}, v_{\text {rest }}\right)={ }_{S}(t ; v())$. This difference was quantified by the following cost functional

$$
\mathcal{J}(v(\quad))=\frac{1}{2} \quad{ }_{0}^{T}\left\|{ }_{R}(t)-{ }_{S}(t ; v(\quad))\right\|^{2} d t
$$

and thus, our optimization problem can be stated as the minimization of (9) subject to both equation (5), and its initial conditions hold. That is,

$$
\begin{aligned}
& \min \mathcal{J}(v()) \quad \text { subjet to } \\
& \frac{v}{t}-D \cdot(v)-J_{T C}=0 \text { in } H, \\
& \frac{v}{\mathbf{n}}=0 \text { on } \quad H, \\
& v-v_{\text {rest }}=0 \text { at } t=0,
\end{aligned}
$$

To solve (10) we used an iterative gradient descent method following an adjoint formulation. Further details can be found in $[8,10,12]$.

\subsection{Tikhonov regularization}

We compared our inverse methodology with zero-order Tikhonov regularization, which is formulated as

$$
\min _{\mathbf{v}(t)}\|(t)-\mathbf{A v}(t)\|^{2}+{ }^{2}\|L \mathbf{v}(t)\|^{2}
$$

where $L=I$ is the identity matrix (in case of zero-order Tikhonov), and is the regularization parameter which was selected according to the L-curve method [13] using function $l_{-}$corner function from the Regularization tools package [14].

For the Tikhonov reconstruction, we set the time point equal to $t=50 \mathrm{~ms}$. Ischemic regions were estimated from the obtained value of $v(t)$. If the $v(t)$ at a certain node was below a threshold value, the node was considered ischemic.

\section{Results}

A circular ischemic region of radius $12 \mathrm{~mm}$ on the cardiac surface was considered, as shown in figure $1(b)$. Then, the forward model was solved and BSPMs were corrupted by AWGN with different SNRs. In order to evaluate the inverse solutions, a binary output was considered (healthy/ischemic) as presented in figure 2 , and the following metrics were used [6,7]: (1) the correlation coefficient (CC) between the real and the reconstructed configuration; (2) the sensitive error ( $\mathrm{SN}$ ), which is defined as the rate of true ischemia that is not detected by the algorithm (false negative); and (3) the specificity error (SP), which is defined as the rate of the misjudged ischemic region out the reconstructed ischemic region (false negative).

In the case of the level set inverse procedure, the iterative method was initialized assuming healthy conditions for the entire tissue. At each iteration, the functional cost gradient was computed and both parameters, ${ }_{i n}\left(\mathbf{r}_{H}\right)$ and $v_{\text {rest }}\left(\mathbf{r}_{H}\right)$, were updated [12]. After 23 iterations, the stop criterion (stationary cost functional) was accomplished providing the final reconstruction. The reconstruction algorithm was applied to a single cardiac cycle of length $T=240 \mathrm{~ms}$ in the steady state.

Overall, the proposed methodology achieved satisfying results. Table 1 shows the quality metrics for both inverse procedures under analysis. In the level set approach, CC exceeded the value of 0.73 for different SNRs values. Similarly, a SN and a SP ratios less than 0.07 and 0.40 were obtained, respectively. In the case of Tikhonov, the correlation coefficient values were not higher than 0.57 .

An example of reconstruction can be seen in Figure 2. Panel (a) correspond to the ground truth ( ${ }_{\text {in }}\left(\mathbf{r}_{H}\right) \quad 0.35 \mathrm{~ms}$ ), were the ischemic zone is represented in red and the healthy region is represented in blue. Panel (b) and (c) shows final reconstructions of the level set method and the zero-order Tikhonov algorithm for $\mathrm{SNR}=20 \mathrm{dBs}$, respectively.

Table 1. Comparative quality metrics

\begin{tabular}{|c|c|c|c|c|c|c|}
\hline & \multicolumn{3}{|c|}{ Tikhonov } & \multicolumn{3}{c|}{ Level Set } \\
\hline \hline SNR & CC & SP & SN & CC & SP & SN \\
\hline 5 & 0.45 & 0.55 & 0.49 & 0.73 & 0.40 & 0.07 \\
\hline 10 & 0.51 & 0.50 & 0.45 & 0.80 & 0.28 & 0.08 \\
\hline 15 & 0.53 & 0.52 & 0.33 & 0.78 & 0.29 & 0.10 \\
\hline 20 & 0.57 & 0.51 & 0.36 & 0.81 & 0.26 & 0.11 \\
\hline
\end{tabular}

\section{Conclusions}

In this work, we extended the methodology proposed in [8] to 3D realistic geometries. We tested our approach with BSPMs corrupted with different SNRs values, and we 


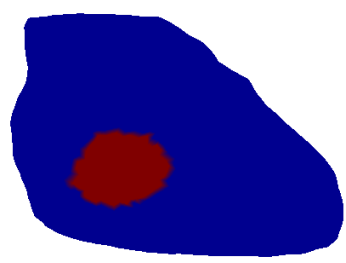

(a) Ground thruth

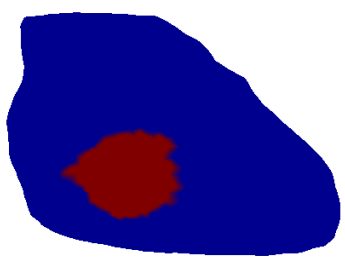

(b) Level-set method

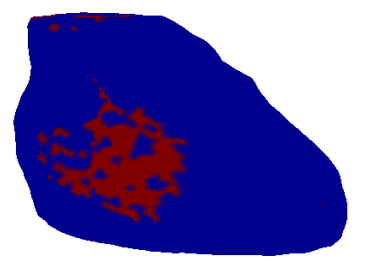

(c) zero-order Tikhonov

Figure 2. Reconstruction of an ischemic region in a real anatomical geometry for $\mathrm{SNR}=20 \mathrm{~dB}$. (a) Representations of the true ischemic region. (b) Reconstruction for the level set approach. (c) Tikhonov reconstruction.

compared it with classical Tikhonov regularization. Our method demonstrated to be superior for all SNRs, reconstructing and locating the ischemic region satisfactorily. In a future work, we aim to incorporate more realistic settings using an electrophysiology detailed model, with a physiological representation of the ischemia model. We will also investigate the performance of this method in localizing ischemia from real clinical measurements. This assumes that the electrophysiological model that would be used to constrain the minimization problem is sufficiently accurate.

\section{Acknowledgments}

This work was supported in part by Spanish MINECO grants TEC-2013-46067-R, FIS2013-41802-R and by Carlos III of Madrid University PIF grant to Carlos E. Chavez.

\section{References}

[1] Gulrajani RM. The forward and inverse problems of electrocardiography. IEEE Engineering in Medicine and Biology Magazine Sep. 1998;17(5):84-101.

[2] Milanic M, Jazbinsek V, MacLeod RS, Brooks DH, Hren R. Assessment of regularization techniques for electrocardiographic imaging. Journal of Electrocardiology 2014; 47(1):20 - 28. ISSN 0022-0736.

[3] Cluitmans M, Peeters R, Westra R, Volders P. Noninvasive reconstruction of cardiac electrical activity: update on current methods, applications and challenges. Netherlands Heart Journal 2015;23(6):301-311.

[4] Li G, He B. Non-invasive estimation of myocardial infarction by means of a heart-model-based imaging approach: a simulation study. Med Biol Eng Comput Jan. 2004; 42(1):128-136. ISSN 0140-0118 (Print); 0140-0118 (Linking).

[5] Nielsen B, Lysaker M, Grottum P. Computing ischemic regions in the heart with the bidomain model; first steps towards validation. IEEE Transactions on Medical Imaging June 2013;32(6):1085-1096.

[6] Wang D, Kirby RM, MacLeod RS, Johnson CR. Inverse electrocardiographic source localization of ischemia: An optimization framework and finite element solution. Journal of Computational Physics 2013;250(0):403 - 424.

[7] Potyagaylo D, Cortés E, Schulze W, Dössel O. Binary optimization for source localization in the inverse problem of ecg. Medical Biological Engineering Computing 2014; 52(9):717-728.

[8] Álvarez D, Alonso-Atienza F, Rojo-Álvarez JL, GarcíaAlberola A, Moscoso M. Shape reconstruction of cardiac ischemia from non-contact intracardiac recordings: A model study. Mathematical and Computer Modelling 2012; $55: 1770-1781$.

[9] Mitchell CC, Schaeffer DG. A two-current model for the dynamics of cardiac membrane. Bulletin of Mathematical Biology 2003;65(5):767 - 793 .

[10] Chávez C, Alonso-Atienza F, Alvarez D. Avoiding the inverse crime in the inverse problem of electrocardiography: estimating the shape and location of cardiac ischemia. In IEEE Computing in Cardiology. ISSN 2325-8861, Sept 2013; 687-690.

[11] Trénor B, Romero L, Ferrero JoséMaría J, Sáiz J, Moltó $\mathrm{G}$, Alonso JM. Vulnerability to reentry in a regionally ischemic tissue: A simulation study. Annals of Biomedical Engineering 2007;35(10):1756-1770.

[12] Chávez CE, Zemzemi N, Coudière Y, Alonso-Atienza F, Álvarez D. Inverse problem of electrocardiography: Estimating the location of cardiac ischemia in a $3 \mathrm{~d}$ realistic geometry. In Functional Imaging and Modeling of the Heart. Springer International Publishing, 2015; 393-401.

[13] Hansen PC. Rank-deficient and discrete ill-posed problems: numerical aspects of linear inversion, volume 4. Siam, 1998.

[14] Hansen PC. Regularization tools version 4.0 for matlab 7.3. Numerical algorithms 2007;46(2):189-194.

Address for correspondence:

Carlos E Chávez

E.P.S Universidad Carlos III de Madrid,

28943, Leganés, Madrid, Spain

cchavez@ing.uc3m.es 